\title{
Sarcoidosis and cancer revisited: a long-term follow-up study of 555 Danish sarcoidosis patients
}

\author{
F.K. Rømer*+, P. Hommelgaard*, G. Schou*
}

Sarcoidosis and cancer revisited: a long-term follow-up study of 555 Danish sarcoidosis patients. F.K. Rømer, P. Hommelgaard, G. Schou. (OERS Journals Ltd 1998.

ABSTRACT: For more than 20 yrs it has been debated whether the systemic disease sarcoidosis predisposes to malignant neoplasms. The aim of this study was to examine the occurrence of cancer in Danish sarcoidosis patients observed for 9-30 yrs.

The clinical data of 555 consecutive sarcoidosis patients were linked with the nationwide Danish Cancer Registry in a database, comparing the results with the expected incidence in the Danish population, adjusted for sex, age and calendar time. The sarcoidosis patients were diagnosed in two areas during the periods of 1960-1971 and 1970-1981, respectively, and followed until December 31, 1991.

No excess of cancer was observed in sarcoidosis patients. A total number of 48 patients were observed with cancer (20 males and 28 females). Cancer occurred 1-29 yrs after sarcoidosis diagnosis (median $14 \mathrm{yrs})$ The observed versus expected (O/E) ratio was 1.16 (95\% confidence interval (CI) 0.75-1.79) in males and 1.28 (95\% CI 0.88-1.86) in females. No increased occurrence of lung cancer or malignant lymphoma $(\mathrm{O} / \mathrm{E}$ ratios $0.23,95 \% \mathrm{CI} 0.00-1.25$ and $1.25,95 \% \mathrm{Cl} 0.02-6.95$, respectively) was found. Neither age at diagnosis of sarcoidosis nor clinical sarcoidosis features were indicators of later occurrence of malignancy.

The study could not confirm previous reports of an increased occurrence of malignant neoplasms in Danish sarcoidosis patients.

Eur Respir J 1998; 12: 906-912.
*Dept of Medicine, Silkeborg Central Hospital, Silkeborg Denmark. +Dept of Respiratory Medicine, Aarhus University Hospital, Aarhus, Denmark. "sanish Cancer Society, Institute of Cancer Epidemiology, Copenhagen, Denmark.

Correspondence: F.K. Rømer, Dept of Medicine, Silkeborg Central Hospital, DK8600 Silkeborg, Denmark

Fax: 4587222357

Keywords: Malignancy, neoplasm, sarcoidosis

Received: May 271997

Accepted after revision June 301998

Preliminary results were presented at the Joint Meeting of the World Association of Sarcoidosis and Other Granulomatous Disorders and British Society for Allergy and Clinical Immunology, London, October 1995 .
Sarcoidosis is a systemic disorder of unknown aetiology, characterized by mononuclear cell infiltrates and granuloma formation, most commonly with clinical signs of disease in the lymph nodes and lungs, although almost any organ may be involved. Most cases run a self-limiting course, but in some patients progressing disease or fibrosis occurs which may become invalidating or lethal [1]. Sarcoidosis can imitate other disorders, especially malignant neoplasms and, conversely, local sarcoid reactions are ob-served in cancer disorders [2].

A possible causal relationship between the benign multisystem granulomatous disorder sarcoidosis and malignant neoplasms has been discussed for about $25 \mathrm{yrs}$ [2]. In 1972 a Danish analysis of about 1,500 cases of malignant lymphoma disclosed five patients who also had sarcoidosis [3], a high rate in view of a previously reported annual sarcoidosis incidence in Denmark of 5/100,000 population [4]. In concordance with this observation, a subsequent registry study showed a higher prevalence than expected of malignant neoplasms among patients reported to a nationwide Danish Sarcoidosis Registry [4] in the period of 1962-1971 [5]. This study has since been widely cited in the literature, although the validity of the results was challenged in a later evaluation of the same series, mainly because the correctness of diagnosis was questioned in several of the cases, thus invalidating the results [6].

Apart from the above-mentioned Danish study, no longitudinal sarcoidosis series specifically dealing with this subject have been available at this point. Because of the discrepancies between the results [5, 6], a long-term followup was initiated in 1980 [7] to examine the occurrence of malignant neoplasms in a consecutive hospital-based series of sarcoidosis patients, using the compulsory nationwide notification to the Danish Cancer Registry. An evaluation was also made as to whether characteristic clinical sarcoidosis manifestations may be associated with an increased risk of cancer.

\section{Methods}

\section{General description of sources}

The material was taken from two sources, as outlined in table 1 (columns 1 and 3). The first source is from the mainly rural Ringkøbing County in western Jutland and included all consecutive cases of sarcoidosis in the period 1960-1969, using files from all hospitals and lung clinics in the region. The study was made in accordance with the thesis of Selroos [10] from 1969 on Finnish sarcoidosis patients [10] and the seminal publications of МАҮоск et al. [11] and S Slтzвасн [12]. The age- and sex-correlated incidence figures were calculated in co-operation with the late O. Horwitz, chief of the Danish Sarcoidosis Registry. The study was initiated because of an impression that sarcoidosis was a rather common disease in the area, which 
Table 1. - Clinical data on 555 sarcoidosis patients from two Danish areas, diagnosed in 1960-1981

\begin{tabular}{|c|c|c|c|c|c|}
\hline & $\begin{array}{c}1 \\
\text { RA } 1960-1969\end{array}$ & $\begin{array}{c}2 \\
\text { RA 1960-1971 }\end{array}$ & $\begin{array}{c}3 \\
\text { Aarhus 1970-1979 }\end{array}$ & $\begin{array}{c}4 \\
\text { Aarhus 1970-1981 }\end{array}$ & $\begin{array}{c}5 \\
1960-1981\end{array}$ \\
\hline Patients $n$ & 238 & 258 & 243 & 297 & 555 \\
\hline Basic population $n$ & 220,000 & & 245,000 & & 465,000 \\
\hline Annual incidence per 100,000 population & 10.8 & & 9.9 & & \\
\hline Urban/rural \% & $35 / 65$ & & $100 / 0$ & & \\
\hline \multicolumn{6}{|l|}{ Age yrs } \\
\hline Median & ND & 29 & ND & 27 & 28 \\
\hline Range & $13-84$ & $12-84$ & $16-77$ & $13-78$ & $12-84$ \\
\hline $10-19$ & $35(15)$ & $38(15)$ & $14 \quad(6)$ & $23 \quad(8)$ & $61(11)$ \\
\hline $20-29$ & $88(37)$ & $95(37)$ & $128(53)$ & $156(53)$ & $251(45)$ \\
\hline $30-39$ & 40 (17) & 44 (17) & $58(24)$ & $62(21)$ & 106 (19) \\
\hline $40-49$ & $27(11)$ & $33(13)$ & $24(10)$ & $31(10)$ & $64(12)$ \\
\hline $50-59$ & 27 (11) & 28 (11) & $10 \quad(4)$ & 13 (4) & $41 \quad(7)$ \\
\hline $60+$ & $21 *(9)$ & $20 *(8)$ & $9 \quad(4)$ & 12 (4) & $32 \quad(6)$ \\
\hline$<40$ & $163(68)$ & $177(69)$ & $200(82)$ & 241 (81) & $418(75)$ \\
\hline Š40 & $75(32)$ & $81(31)$ & 43 (18) & 56 (19) & $137(25)$ \\
\hline Males & $109(46)$ & $114(44)$ & $128(53)$ & $156(53)$ & $270(49)$ \\
\hline Females & $129(54)$ & $144(56)$ & 115 & $141(47)$ & $285(51)$ \\
\hline Detected by routine chest radiography & $\mathrm{ND}$ & $173(67)$ & $118(49)$ & $131(44)$ & $304(55)$ \\
\hline Admission because of symptoms & ND & $85(33)$ & $125(51)$ & $166(56)$ & $251(45)$ \\
\hline \multicolumn{6}{|l|}{ Chest radiography } \\
\hline No abnormalities & $5 \quad(2)$ & $7 \quad(3)$ & $1 \quad(-)$ & $2(1)$ & 9 (2) \\
\hline Stage I & $116(49)$ & $134(52)$ & $135(56)$ & $181(61)$ & $315(57)$ \\
\hline Stage II & $94(39)$ & $99(38)$ & $96(40)$ & $98(33)$ & $197(35)$ \\
\hline Stage III & $23(10)$ & $18 \quad(7)$ & 11 (5) & $16 \quad(5)$ & 34 (6) \\
\hline Erythema nodosum/Löfgren's syndrome & $47(20)$ & $58(22)$ & 35 (14) & $53(18)$ & $111(20)$ \\
\hline Other extrathoracic manifestations ${ }^{\dagger}$ & $\mathrm{ND}$ & $75(29)$ & $50(21)$ & $90(30)$ & $165(30)$ \\
\hline Biopsy-verified & $178(75)$ & $201(78)$ & $106(44)$ & $111(37)$ & $312(56)$ \\
\hline Clinical/chest radiographic diagnosis & $60(25)$ & $57(22)$ & $137(56)$ & $186(63)$ & $243(44)$ \\
\hline Systemic steroid treatment & ND & $63(24)$ & $\mathrm{ND}$ & 42 (14) & 105 (19) \\
\hline Vital status at end of follow-up period & ND & $\mathrm{ND}$ & $\mathrm{ND}$ & ND & \\
\hline Dead & & & & & $91(16)$ \\
\hline Emigrated & & & & & 9 (2) \\
\hline Alive & & & & & $455(82)$ \\
\hline
\end{tabular}

Column 5=columns 2+4. Data from the previously published series from 1960-1969 (Ringkøbing area (RA)) [8] and 1970-1979 Aarhus City [9] are given in columns 1 and 3, respectively, and data from the extended and revised series from the two areas are shown in columns 2 and 4, which are combined into the total series. Data are shown as n (\%). ND: no data are available or are given in another way in the original communications; age is at onset or diagnosis of sarcoidosis. *: correction for one patient who was placed in a wrong age group in the original paper [8]. ${ }^{\dagger}$ : Eyes, skin, peripheral lymph nodes, liver, spleen, gastrointestinal, musculoskeletal, joints, heart, lacrimal glands, central nervous system and peripheral nerves, calcium abnormalities, kidneys, genitourinary, pleura.

could be in accordance with the theory, which was very popular at that time, on an association between pine pollen and sarcoidosis. The primary aim was to evaluate the incidence and clinical manifestations of sarcoidosis in the area. A total of 238 new cases of sarcoidosis was observed, corresponding to an annual incidence of 10.8/ 100.000 population (table 1 , column 1 ), a figure twice that formerly reported for Denmark [4]. An incidental observation was the existence of a large deficit of notification to the Sarcoidosis Registry because $31 \%$ of the patients were not reported to the registry; therefore, the general results from the Sarcoidosis Registry could be doubted if underregistration was a common phenomenon. The results were published in 1973 [8].

The second source is from the Aarhus City area, where a similar study was made, covering the period 1970-1979 (table 1, column 3). This series may not be as complete as the former; a few patients with predominate extrathoracic sarcoidosis diagnosed at specialized departments may have been missed (e.g. ophthalmology, otorhinolaryngology, neurology), although it was a general rule that every patient with sarcoidosis should be referred to the Chest Clinic. A total of 243 newly diagnosed patients was record- ed from the files of the Chest Clinic and the University Department of Medicine, corresponding to an annual incidence of 9.9/100,000 population, which was comparable to the figures from the Ringkøbing area. Of these 243 patients, 210 were included in a prognosis study. The results were published in 1982 [19].

After these studies were completed, new patients were recorded consecutively for the following 1-2 yrs in each area; in Ringkøbing County a further 43 cases were noted in 1970-1971 (i.e. 281 new cases in 1960-1971), and in Aarhus City area 56 cases in 1980-1981 (i.e. a total of 299 new cases in 1970-1981). The series were followed up regularly during 1970-1988, when minor corrections (e.g. errors in the year of diagnosis or in age) were necessary. In addition, a few patients were detected who had actually been diagnosed in the preceding decade in question. Therefore, after the inclusion of 99 new cases, clinical data were available on a total of 580 sarcoidosis patients from the two areas, diagnosed in the period 19601981.

All patients were Caucasian and were born in Denmark. No consistent information on smoking habits was available. 


\section{Diagnosis}

The diagnosis of sarcoidosis was made according to common practice in Scandinavia [10] and in accordance with SiLтzвасн [12]. The diagnosis was based upon appropriate clinical and chest radiographic features and, in many cases, by the demonstration of noncaseous epithelioid cell granulomas, occurring in patients in whom other causes (e.g. tuberculosis and other infections, neoplasia) could be excluded.

Many patients had a typical clinical picture, including Löfgren's syndrome, uveitis, etc., together with chest radiographic changes. The radiographs were examined by the same teams of experienced chest physicians or radiologists and each radiograph was examined independently by at least two physicians. All patients were examined according to routine practice in the departments in question. Before inclusion in the present series the patient records, etc., were critically evaluated several times by one of the authors (F.K. Rømer) and ad hoc working groups, and no cases were included before an observation period for at least 6 months with repeated radiographic and clinical examinations.

The finding of enlarged hilar lymph nodes at one occasion in an asymptomatic patient without histological evidence of sarcoidosis was, therefore, not accepted as a reason for inclusion. The abnormality should be reproduced at the following visits. The observation period also prevented inclusion of cases with nonsarcoid conditions, e.g. infections and malignancies.

\section{Patient data and identification}

The hospital files of all 580 sarcoidosis patients were carefully re-evaluated, and demographic and clinical data, including symptoms, chest radiographic and other paraclinical examinations, including results of histology, bacteriology and information on further course were obtained during follow-up.

For the present study information on cancer and death was also required. However, a complication was that in the majority of the older files the patients were identified only by name, date and place of birth, and home address at admission, together with a local file number. This was common practice in Danish hospitals for years, but it was insufficient for the present study.

Since 1968 each Danish inhabitant has been assigned a unique Central Person Registration (CPR) number which permitted an unequivocal, complete identification of every individual in the kingdom. In co-operation with the Danish National Central Person Registry, in 1990-1991 the CPR for the patients and information on possible emigration were obtained. The Danish Cancer Registry has registered cancers on a national basis since 1943 [13]. Since 1987 , reporting to this registry of all new cases of malignant neoplasms has been mandatory for all hospitals, clinics and physicians. Cancer was classified according to the International Classification of Diseases (ICD)7 codes [13]. By means of the CPR number, relevant information on cancer was obtained from the Danish Cancer Registry. From the computerized files of the national Death Registry the date of death for patients was obtained, again by means of the CPR number. The final analysis was made in April 1995, covering the period from January 1, 1960 to December 31, 1991.

\section{Statistics}

For each patient the time of entry was date (yr) of diagnosis or onset of sarcoidosis. The time of exit was the date of the first of the following events: cancer, emigration, death or the closing date, December 31, 1991. Patients with cancer (except for skin cancer) before sarcoidosis were excluded.

In this cohort design the observed incidence $(\mathrm{O})$ was compared with the expected incidence $(\mathrm{E})$, as calculated from the national cancer incidence rates, taking account of the sex, age and calendar time, and O/E ratios (or standardized mortality rates (SMR)) were calculated [14]. Age and calendar time were grouped in 5-yr intervals. The effect of covariates was evaluated by stratification. The person-yr calculations were made using the Person Years (PYRS) program [15]. Confidence intervals (CI) are Byars limits [16].

The study was conducted in accordance with the Helsinki Declaration, as approved by the local ethical committee. The linkage of data from the public registers was approved by the Danish National Data Protection Agency.

\section{Results}

\section{Patients}

From the 580 cases 25 subjects were excluded because they could not be unambiguously identified; they were 23 from the Ringkøbing series and two from Aarhus. However, because their demographic and clinical data were not different from those of the remaining patients (e.g. age range 13-73 yrs median age $30 \mathrm{yrs}$ ), their exclusion from the series should not have severely altered the results of the study.

The final series thus consisted of 555 patients who had the diagnosis of sarcoidosis in the period 1960-1981. There were 270 males and 285 females (age at diagnosis 12-84 yrs, median age $28 \mathrm{yrs}$ ). The most important clinical data of the patients are outlined on table 1 , which shows final data from the Ringkøbing area and Aarhus in columns 2 and 4, respectively. The cumulated data in all 555 cases are given in column 5.

\section{Cancer}

Among the 555 sarcoidosis patients a total number of 48 cases of malignancy occurred in a follow-up time of 9 31 yrs, for males 4,987 person-yrs and for females 5,408 person-yrs.

The cases with malignancy included 20 males and 28 females. (It is a curious coincidence that this is the same figure as the number of patients with sarcoidosis and cancer, reported in 1974 by BRINCKER and WILBEK [5].) These figures were 1.16 and 1.28 times the expected number of cases, respectively. However, these $\mathrm{O} / \mathrm{E}$ ratios were not statistically significant (table 2 ).

Sarcoidosis patients with subsequent cancer had a median age of $48 \mathrm{yrs}$ at diagnosis of sarcoidosis (range 19-78 yrs), compared with 28 yrs in the whole cohort. The median age at cancer diagnosis was 64 yrs (range 30 80 ). However, patients older than 50 yrs at diagnosis of sarcoidosis had only a slightly increased risk of subsequent cancer compared with younger patients (in males, 
Table 2. - Observed $(\mathrm{O})$ versus expected $(\mathrm{E})$ number of malignancies, by sex and latency (yrs) from sarcoidosis to malignant disease with $95 \%$ confidence intervals $(\mathrm{Cl})$

\begin{tabular}{llrccc}
\hline Sex & Latency & O & E & O/E & 95\% CI \\
\hline Males & & & & & \\
& $0-9$ & 4 & 5.92 & 0.68 & $0.18-1.73$ \\
& $10-19$ & 9 & 7.30 & 1.23 & $0.56-2.34$ \\
& Š20 & 7 & 4.09 & 1.71 & $0.68-3.52$ \\
& Total & 20 & 17.3 & 1.16 & $0.75-1.79$ \\
Females & & & & & \\
& $0-9$ & 10 & 7.30 & 1.37 & $0.66-2.52$ \\
& $10-19$ & 10 & 9.34 & 1.07 & $0.51-1.97$ \\
& Š20 & 8 & 5.22 & 1.53 & $0.66-3.02$ \\
& Total & 28 & 21.9 & 1.28 & $0.88-1.86$ \\
\hline
\end{tabular}

the $\mathrm{O} / \mathrm{E}$ ratio was 1.09 , in those aged $<50 \mathrm{yrs}$, compared with 1.22 in those aged Š50 yrs; in females, these O/E ratios were 1.12 and 1.64 , respectively; these ratios were not statistically different from 1.0).

Although the total number of malignancies was not increased, it was also evaluated whether there existed a preponderance of certain types of cancer. The cancer types (ICD7 codes) are listed in table 3 . None of these figures exceeded the $95 \% \mathrm{CI}$ and the pattern was not unusual, although the occurrence of only one case of lung cancer may be surprising.

Of interest may be the one case of malignant lymphoma observed in a 78 yr-old female with lymphoreticulosarcoma of the stomach, occurring 20 yrs after stage II pulmonary sarcoidosis with demonstration of typical noncaseous epithelioid cell granulomas by Daniels' biopsy. No autopsy report was available.

\section{Time delay (latency) between sarcoidosis and cancer}

The interval from the diagnosis of cancer was 1-29 yrs (median $14 \mathrm{yrs}$ ). The risk of cancer was slightly increased with time since onset or diagnosis of sarcoidosis, but not significantly increased in any of the intervals 0-9, 10-19 or S20 yrs after sarcoidosis, compared with the reference population (table 2). Likewise, there were no consistent differences when the most frequent types of cancer were analysed separately, although the number of patients in these subgroups was extremely small (table 1).

\section{Specific sarcoidosis manifestations and cancer}

Analysis of subgroups could not demonstrate any increased risk of cancer dependent on certain sarcoidosis manifestations, i.e. age, chest radiographic stages, presence of erythema nodosum or available histological confirmation of diagnosis. For example, among 111 patients with erythema nodosum, associated with an excellent spontaneous prognosis [9], malignant neoplasia occurred in eight $(7.2 \%)$, compared with 40 cases $(9.0 \%)$ in 446 patients without erythaema nodosum. Furthermore, no consistent pattern was observed concerning patients with or without steroid treatment.

\section{Discussion}

In the present study no increased risk of malignant disease in sarcoidosis could be demonstrated. Nor could any peculiarities in sarcoidosis be found indicating specific predispositions or risk factors. The patients with cancer had a higher age at sarcoidosis diagnosis than those without cancer, but this may mirror the generally increased cancer rate in older persons. All hospital records were critically evaluated, so the sarcoidosis diagnosis was reasonably certain in all cases. Likewise, the reporting to the Cancer Registry is carefully controlled in several steps [13].

Table 3. - Observed neoplasms (O) in 555 sarcoidosis patients, compared with the expected number (E)

\begin{tabular}{|c|c|c|c|c|c|}
\hline \multirow[t]{2}{*}{ ICD7 code } & \multirow[t]{2}{*}{ Localization } & \multicolumn{2}{|c|}{ Number of patients } & \multirow[t]{2}{*}{$\mathrm{O} / \mathrm{E}$} & \multirow[t]{2}{*}{$95 \% \mathrm{CI}$} \\
\hline & & $\mathrm{O}$ & $\mathrm{E}$ & & \\
\hline $140-144$ & Lip and mouth & 3 & 0.58 & 5.17 & $1.04-15.11$ \\
\hline 151 & Stomach & 2 & 1.18 & 1.69 & $0.19-6.12$ \\
\hline $153+253$ & Colon & 6 & 2.53 & 2.37 & $0.87-5.16$ \\
\hline 154 & Rectum & 2 & 1.55 & 1.29 & $0.14-4.66$ \\
\hline $155+156$ & Liver & 3 & 0.48 & 6.25 & $1.26-18.26$ \\
\hline 162 & Lung & 1 & 4.44 & 0.23 & $0.00-1.25$ \\
\hline 170 & Female breast & 6 & 5.60 & 1.07 & $0.39-2.33$ \\
\hline $171-176$ & Female genital organs & 3 & 4.36 & 0.69 & $0.14-2.01$ \\
\hline 177 & Prostate & 4 & 1.42 & 2.82 & $0.76-7.21$ \\
\hline 178 & Testis & 1 & 0.68 & 1.47 & $0.02-8.18$ \\
\hline 180 & Kidney & 1 & 0.98 & 1.02 & $0.01-5.68$ \\
\hline 190 & Melanoma & 4 & 1.27 & 3.15 & $0.85-8.06$ \\
\hline 191 & Other skin neoplasms & 9 & 4.57 & 1.97 & $0.90-3.74$ \\
\hline 199 & Unspecified site & 1 & 0.29 & 3.45 & $0.05-19.19$ \\
\hline 200,202 & $\begin{array}{l}\text { Non-Hodgkin's lymphoma } \\
\text { (Lymphoreticulosarcoma of the stomach) }\end{array}$ & 1 & 0.80 & 1.25 & $0.02-6.95$ \\
\hline 204 & Lymphatic leukaemia & 1 & 0.85 & 1.18 & $0.02-6.55$ \\
\hline \multicolumn{6}{|c|}{ Relevant malignancies which were not observed in the series: } \\
\hline 201 & Hodgkin's disease & 0 & 0.31 & & \\
\hline 203 & Multiple myeloma & 0 & 0.34 & & \\
\hline 205 & Mycosis fungoides & 0 & 0.02 & & \\
\hline
\end{tabular}

ICD: International Classification of Diseases. 
As reviewed recently [2], the association between sarcoidosis and malignancy has several aspects. The clinical and radiographic pictures of sarcoidosis and malignancy may mimic each other, and local sarcoid reactions are well known in the vicinity of neoplasms, most frequently noticed in bronchial carcinoma [17] and malignant lymphomas [18], making the distinction from coexistent sarcoidosis difficult in some cases. A unique example is the occurrence of sarcoidosis in patients treated for testicular carcinoma [19, 20], with 48 cases being reported in 16 studies [21]. This may be explained by the combination of young age, an excellent prognosis of testicular cancer and frequent chest radiographic examinations for several years [19].

Besides several theories on pathogenic links and immunological predisposition in cases with both sarcoidosis and malignancy [21-24], a practical consequence is the considerable risk of misdiagnosis, leading to wrong treatment. In particular, the risk of overlooking a malignant disease is feared among the physicians who manage sarcoidosis patients, although this risk is very small, at least in asymptomatic bilateral hilar adenopathy, on the condition that a careful observation is undertaken [25]. The diagnostic dilemma is excellently demonstrated by BOGAERTS et al. [26].

As mentioned above, the study by BRINCKER and WLBEK [5] suggested an increased incidence of cancer in Danish sarcoidosis patients, linking data from the Danish Cancer Registry and a registry of newly diagnosed respiratory sarcoidosis, based on voluntary reporting from the chest clinics during the years 1962-1971 [4]. Among 2,544 patients who were notified in the sarcoidosis registry the total number of patient with cancer after diagnosis of sarcoidosis was 48, against 33.8 expected cases. Malignant lymphomata were reported to occur 11 times more than expected $(\mathrm{O} / \mathrm{E}$ ratio $6 / 0.5)$ and lung cancer three times more than expected (O/E ratio 9/2.8), while the occurrence of other neoplasms was not increased. An important point was that the patient data were collected from the notification files only, without reviewing the hospital records, because the authors felt that "a rejection of the diagnosis in one or more of these cases would merely result in a statistically unacceptable alteration in the basis for the calculations" [5]. The clinical and demographical data of the study were almost on cancer, whereas data concerning sarcoidosis were sparse.

With the primary aim of demonstrating any possible common features characterizing sarcoidosis patients who later developed a malignant disease, RøMER [6] reviewed, with permission from the authors, the chest clinic and hospital records of the 48 patients who were reported to have sarcoidosis and subsequent cancer, surprisingly disclosing several notification errors. A total of 14 cases $(29 \%)$ could be excluded because they did not fulfil the criteria of cancer (three cases) or sarcoidosis (10 cases) or had cancer before sarcoidosis (one case); only 34 cases were left in which it was likely that cancer occurred in patients with proven sarcoidosis, a figure which was exactly as expected (i.e. 33.4 cases). As a consequence, both the results and methodology of the study by BRINCKER and WLBEK [5] were heavily challenged at the 1978 International Sarcoidosis Congress [6].

BRINCKER and WILBEK [5] argued for no case-by-case evaluation in their study. However, the argument of the present authors was that a reduction in the total number of sarcoidosis patients, corresponding to a $20 \%$ reduction in the number of patients in the original study (10 of 48 cases did not have sarcoidosis), could not be extended to the sarcoidosis registry as a whole, because the group with sarcoidosis and cancer was not a random group; indeed, it might be considered a very special group of patients with inherent diagnostic difficulties. Furthermore, it was known that the failure rate of notification to the sarcoidosis registry was up to $31 \%$ [8], suggesting that the estimation of the annual incidence of sarcoidosis of 5/100,000 in Denmark [4] was too low. Based on consecutive hospital and chest clinic records from Ringkøbing County and the Aarhus area, the annual incidence was found to be about $10 / 100,000$ population $[8,9]$.

Because the former sarcoidosis registry (and, therefore, also a combined registry study) might not be representative for the Danish sarcoidosis population as a whole, and because the previous results [5] were based on a series where $29 \%$ of the patients did not fulfil the criteria of sarcoidosis followed by cancer, the present study was initiated in co-operation with the Danish Cancer Registry in order to examine the occurrence of cancer in a consecutive and complete as possible series of sarcoidosis patients from all hospitals and chest clinics in the two regions in question. From the results it is evident that the conclusions of BRINCKER and WLLBEK [5] could not be confirmed.

The present study has some limitations. One is that the "true" incidence of sarcoidosis in the population is virtually unknown. Many cases run a symptomless and selflimiting, course and are never diagnosed. The size of this group of silent sarcoidosis is unknown, but the results from a Swedish autopsy study suggested that the prevalence of sarcoidosis may be up to 10 times more frequent than generally believed [27]. At present, the number of undetected sarcoidosis patients may be growing further after cessation of chest radiographic screening for pulmonary tuberculosis. Another point is that sarcoidosis in this part of the world generally has a very mild course, compared with the disease elsewhere, including a high incidence of the benign Löfgren's syndrome with erythema nodosum and bilateral hilar adenopathy as hallmarks [28]. Therefore, these results may not be generalizable to other parts of the world.

The most severe objection to the present results may be that they were based on series with a high proportion of patients without histological confirmation of the diagnosis, i.e. demonstration of the characteristic noncaseating epithelioid granulomas. However, both Löfgren's syndrome and the radiographic features of sarcoidosis were familiar and all patients were followed for 1-2 yrs or more. Biopsy was performed only in selected cases. If the series had only included patients with a positive biopsy, it may not be representative, with a risk of bias towards more severe cases.

The cancer incidence was independent of several indices of disease severity. Regrettably, the subgroup with severe chronic fibrotic pulmonary sarcoidosis (a group which may be associated with an increased risk of lung cancer [29]) was too small for independent statistical analysis.

The present results are in accordance with the common experience among clinicians managing sarcoidosis and also with published studies on the course and prognosis of sarcoidosis, although cancer was not especially looked for [30, 31] as well as a Danish sarcoidosis study by MunKGAARD 
[32], where the cancer occurrence was mentioned incidentally.

Most importantly, during the preparation of the present manuscript, another Danish study with a similar design reported on results which are in agreement with those of the present study [33]. In that study, 254 sarcoidosis patients from Copenhagen, diagnosed in the period of 19521970, were linked with the Danish Cancer Registry and followed until December 31, 1992, with respect to cancer occurrence. Apart from a higher proportion of biopsy-verified cases $(76 \%)$ and stage III disease $(27 \%)$, the clinical and demographic data were very similar to those of the present study.

Although the observed incidence of lung cancer was twice that expected, it did not exceed the $95 \%$ CI and the overall cancer incidence was not increased. No cases of malignant lymphoma were observed and the results did not support a theory on an association between sarcoidosis and malignancy. The authors concluded that "the main reason other studies have shown such an association is most likely to have been due to selection bias and misclassification".

However, from an oncological point of view, sarcoidosis and malignancy may be associated more frequently than by chance $[21,22]$ and the existence of a "sarcoidosislymphoma syndrome" has been proposed [34], although mainly based on single observations or small series.

A few other studies on series of sarcoidosis patients have obtained varying results. MARSCHKE [35] reported in an abstract on a 30-yr Mayo Clinic expedience of patients with both sarcoidosis and cancer. Among 2,700 cases of sarcoidosis 68 patients with cancer were recorded, with an over-representation of lymphoma and an under-representation of lung cancer. No distinctive features predicting predisposition to cancer were observed in patients with sarcoidosis, but the information on patient data was very sparse. A Japanese questionnaire study [36] reported on increased death rate from lung cancer in a cohort of 1,411 sarcoidosis patients followed for a 3-yr period, but the number of deaths from all cancers was not increased. However, the number of patients with lung cancer was very small (three cases), which may weaken the conclusions. Recently, REICH et al. [37] published a linkage analysis, linking a tumour registry with a sarcoidosis registry of 243 cases observed for 24 yrs. They found evidence that sarcoidosis and malignancy were aetiologically re-lated in about one quarter of cases in which both were present; malignancy was reported in 12 cases, i.e. $4.5 \%$ (against $8.6 \%$ in the present study). None of these cases was a malignant lymphoma or lung cancer. However, for methodological reasons it is difficult to compare their results with others. Therefore, the results of these three studies are conflicting.

In conclusion, with the reservation that geographical or racial differences may exist, the present Danish experience does not confirm a hypothesis of increased cancer risk in sarcoidosis.

\section{References}

1. Newman LS, Rose CS, Maier LA. Sarcoidosis. $N$ Engl $J$ Med 1997; 336: 1224-1234.
2. Rømer FK. Sarcoidosis and cancer. In: James DG, ed. Sarcoidosis and Other Granulomatous Disorders. New York, Marcel Dekker, 1994; pp. 401-415.

3. Brincker H. Sarcoid reactions and sarcoidosis in Hodgkin's disease and other malignant lymphomata. Br J Cancer 1972; 126: 120-128.

4. Horwitz O, Payne PG, Wilbek E. Epidemiology of sarcoidosis in Denmark. Dan Med Bull 1967; 14: 178-182.

5. Brincker H, Wilbek E. The incidence of malignant tumours in patients with respiratory sarcoidosis. Br J Cancer 1974; 29: 247-251.

6. Rømer FK. Sarcoidosis and cancer - a critical view. In: Jones Williams J, Davies BH, eds. Proceedings of the VIIIth International Conference on Sarcoidosis. Cardiff, Alpha Omega Press, 1980; pp. 567-571.

7. Rømer FK. Sarcoidosis and cancer: A prospective study. In: Grassi C, Pozzi E, Rizzato G (eds). Sarcoidosis and Other Granulomatous Disorders. Proceedings of the XI World Congress on Sarcoidosis and Other Granulomatous Disorders, Milan, 6-11 September 1987. Excerpta Medica International Congress Series No. 756; Elsevier Sciences Publishers, 1988; pp. 327-328.

8. Rømer FK, Paulsen S, Antonius V, Nielsen JL, Hommelgaard P. Sarcoidosis in a Danish "Amt" - a retrospective epidemiological study on sarcoidosis in Ringkøbing Amt in the period 1960-1969. Dan Med Bull 1973; 20: 112120.

9. Rømer FK. Presentation of sarcoidosis and outcome of pulmonary changes - a review of 243 patients followed for up to 10 years. Dan Med Bull 1982; 29: 27-32.

10. Selroos $O$. The frequency, clinical picture and prognosis of pulmonary sarcoidosis in Finland. Acta Med Scand 1969; Suppl. 503 (Thesis).

11. Mayock RL, Bertrand P, Morrison CE, Scott JH. Manifestations of sarcoidosis. Analysis of 145 patients, with a review of nine series selected from the literature. Am J Med 1963; 35: 67-89.

12. Siltzbach LE. Sarcoidosis: clinical features and management. Med Clin N Am 1967; 51: 483-502.

13. Storm HH, Pihl J, Michelsen E. Cancer Incidence in Denmark 1992. Copenhagen, Danish Cancer Society, 1996.

14. Clayton D, Hills M. Statistical Models in Epidemiology. Oxford, New York, Tokyo, Oxford University Press, 1993; pp. $147-150$

15. Coleman M, Douglas A, Hermon C, Peto J. Cohort study analysis with a Fortran computer program. Int J Epidemiology 1986; 15: 134-137.

16. Breslow NE, Day NF. Statistical Methods in Cancer Research, Vol. II, Design and Analysis of Cohort Studies. IARC Scientific Publications No. 82. Lyon, International Agency for Research on Cancer, 1987; pp. 69-71.

17. Laurberg P. Sarcoid reactions in pulmonary neoplasms. Scand J Respir Dis 1975; 56: 20-27.

18. Sacks EL, Donaldson SS, Gordon J, Dorfman RF. Epithelioid granulomas associated with Hodgkin's disease. Clinical correlations in 55 previously untreated patients. Cancer 1978; 41: 562-567.

19. Fosså AD, Abeler V, Marton PF, Lien H, Høie J. Sarcoid reaction of hilar and paratracheal lymph nodes in patients treated for testicular cancer. Cancer 1985, 56: 2212-2216.

20. Toner GC, Bosl W. Sarcoidosis, "sarcoid-like lymphadenopathy", and testicular germ cell tumors. Am J Med 1990, 89: 651-656.

21. Pandha HS, Griffiths H, Waxman J. Sarcoidosis and cancer (Editorial). Clin Oncol 1995; 17: 277-278.

22. Brincker H. Coexistence of sarcoidosis and malignant disease: causality or coincidence? Sarcoidosis 1989; 16: 
31-43 (including correspondence Sarcoidosis 1990; 17: 80-82).

23. Karakantza M, Matutes E, MacLennan K, O'Connor NTJ, Srivastava PC, Catovsky D. Association between sarcoidosis and lymphoma. J Clin Pathol 1996; 49: 208-212.

24. Scadding JG. Commentary. Thorax 1996; 51: 448-449.

25. Reich JM, Brouns MC, O'Connor EA, Edwards MJ. Mediastinoscopy in patients with presumptive stage I sarcoidosis. A risk/benefit, cost/benefit analysis. Chest 1998; 113: 147-153.

26. Bogaerts Y, Van der Straeten M, Tasson J, Pauwels R. Sarcoidosis or malignancy: a diagnostic dilemma. Eur $J$ Respir Dis 1983, 64: 541-550.

27. Hägerstrand I, Linell F. The prevalence of sarcoidosis in the autopsy material from a Swedish town. Acta Med Scand 1964; 176: Suppl. 425, 171-173.

28. Sharma OP. Sarcoidosis: Clinical Management. London, Butterworths, 1984.

29. Turner-Warwick M, Lebowitz M, Burrows B, Johnson A. Cryptogenic fibrosing alveolitis and lung cancer. Thorax 1980; 35: 496-499.

30. Johns CJ, MacGregor MI, Zachary JB, Kaplan J, Silverman M. Chronic sarcoidosis: outcome, unusual features and complications. In: Jones Williams J, Davies BH, eds.
Proceedings of the VIIIth International Conference on Sarcoidosis. Cardiff, Alpha Omega Press, 1980; pp. 558-566.

31. Hillerdal G, Nöu E, Osterman K, Schmekel B. Sarcoidosis: epidemiology and prognosis. A 15-year European study. Am Rev Respir Dis 1984; 130: 129-132.

32. Munkgaard S. A follow-up study of patients with intrathoracic sarcoidosis for risk of development of other diseases. In: Chrétien J, Marsac J, Saltiel JC, eds. Proceedings of the IXth International Conference on Sarcoidosis and Other Granulomatous Disorders. Paris, Pergamon Press, 1983; pp. 250-252.

33. Seersholm N, Vestbo J, Viskum K. Risk of malignant neoplasms in patients with pulmonary sarcoidosis. Tho$\operatorname{rax} 1997$; 52: 892-894.

34. Brincker H. The sarcoidosis-lymphoma syndrome. $\mathrm{Br} J$ Cancer 1986; 54: 467-473.

35. Marschke RF. Sarcoidosis and malignant neoplasm: the Mayo Clinic experience. Sarcoidosis 1986; 3: 149-150.

36. Yamaguchi M, Odaka M, Hosoda Y, Iwai K, Tachibana T. Excess death of lung cancer among sarcoidosis patients. Sarcoidosis 1991; 8: 51-55.

37. Reich JM, Mullooly JP, Johnson RE. Linkage analysis of malignancy-associated sarcoidosis. Chest 1995; 107: 605-613. 